\title{
ANTS OF THE GENUS PSEUDOMYRMEX (HYMENOPTERA: FORMICIDAE) FROM DOMINICAN AMBER, WITH A SYNOPSIS OF THE EXTANT ANTILLEAN SPECIES
}

\author{
By PHILIP S. WARD \\ Department of Entomology \\ University of California \\ Davis, CA 95616
}

\section{INTRODUCTION}

The Dominican amber ant fauna is becoming increasingly well known as a result of the recent studies by Baroni Urbani (1980a-d; Baroni Urbani \& Wilson, 1987) and Wilson (1985a-e, 1986, 1988). These investigations reveal a fauna, of probable Oligocene/ early Miocene age (Baroni Urbani \& Saunders, 1982; Krishna \& Grimaldi, 1991; Lambert et al., 1985), containing a diverse array of genera, of which the great majority are extant in the Neotropical region although some have become extinct on Hispaniola. Most of the ant genera are known from only one or two fossil species in Dominican amber. In this paper I report on a genus of arboreal ants, Pseudomyrmex, which has a far richer representation of species in Dominican amber than other genera examined to date. Pseudomyrmex is also an important component of contemporary Neotropical ant communities, so that a detailed comparison of fossil and extant taxa can be attempted. This allows a finer focus on biogeographical changes in the ant fauna, heretofore analyzed largely at the genus level.

I take pleasure in dedicating this paper to William L. Brown, Jr., in appreciation of his generous help on many occasions and the inspiration provided by his benchmark contributions to ant systematics.

\section{METHODS}

The raw material for this study is a series of 30 specimens ( 28 workers, 1 queen, 1 male) of Pseudomyrmex, from 29 pieces of

Manuscript received 24 June 1992 
Dominican amber, about half of which were borrowed from the Museum of Comparative Zoology, Harvard University (MCZC). The specimens have been assigned unique collection numbers (PSW-DA1 to PSW-DA30) and all have been deposited in the MCZC except those originating from the Poinar collection in the Museum of Paleontology, University of California, Berkeley (UCBC). All material was examined in mineral oil, at 25-50× magnification, with a fibre-optic light source.

The following measurements and indices are used (for a more complete explanation of all of these except HD and LCI, introduced here for the first time, see Ward (1989)): HW, head width; HL, head length; HD, head depth: maximum dorsoventral depth of head, measured in lateral view; EL, eye length; OD, ocellar distance; MFC, minimum distance between frontal carinae; SL, scape length; LF1 and LF2, length of the first and second funicular segments, respectively; FL, profemur length; FW, profemur width; DPL, diagonal length of propodeum; BF, length of basal (= dorsal) face of propodeum; DF, length of declivitous face of propodeum; MP, depth of metanotal groove; PL, petiole length; PH, petiole height; PPL, postpetiole length; DPW, dorsal petiolar width; MPW, minimum petiolar width; PPW, postpetiole width; LHT, length of metatibia; CI, cephalic index (HW/HL); LCI, lateral cephalic index (HD/HL); REL, relative eye length (EL/HL); FCI, frontal carinal index $(\mathrm{MFC} / \mathrm{HW})$; SI2, scape index using EL (SL/EL); FI, profemur index (FW/FL); MPI, metanotal index (MP/HW); PLI, petiole length index (PH/PL); PWI, petiole width index (DPW/PL); PWI3, petiole width index, using MPW (MPW/DPW); PPWI, postpetiole width index (PPW/PPL). Measurements were made to the nearest thousandth of a millimeter, but they have been rounded here to the second decimal place. Because of refractive distortion (due to the cut of the amber) or positioning difficulties some measurements are considered rather approximate; these have been prefaced by "ca."

Information on the present-day distribution of Pseudomyrmex species in the West Indies and elsewhere is based on direct examination of material in a large number of collections (see list in Ward, 1989), as part of my ongoing taxonomic research on this genus. For the most part I have not used literature records, 
including citations in Kempf (1972), because these are often unreliable for Pseudomyrmex.

\section{The PSEudomyrmex OF Dominican AMber}

\section{Pseudomyrmex antiquus, new species}

(Figures 1, 12)

Holotype worker: Dominican amber, La Toca mine (\#PSW-DA21) (in amber piece $\mathrm{H}-10-147$, UCBC).

Measurements: HW ca. 1.12, HL ca. 1.21, HD 0.78, EL 0.63, SL 0.55, FL ca. 0.86, FW ca. 0.36, LHT 0.96, DPL 1.17, PL 0.64, PH 0.40, PPL 0.56, DPW ca. 0.37, PPW ca. 0.60

Description: Large species (see measurements). Mandibles with about 6 rather robust teeth on the masticatory margin. Head somewhat quadrate, longer than broad or deep (CI 0.93, LCI 0.64), posterior margin slightly concave, rounding sharply into sides of head. In frontal view median clypeal lobe with a straight anterior margin, rounded laterally. Frontal carinae moderately well separated (MFC $\approx 0.03$ ), fusing anterolaterally with antennal sclerites. Second and third funicular segments about 1.4 times longer than wide. Eyes elongate (REL ca. 0.52), not reaching the level of the median ocellus. Ocellar triangle small $(\mathrm{OD} \approx 0.06)$. Vertex separated from side of head by a weak (broadly rounded) ridge between posterior margin of eye and posterolateral corner of head. Pronotum laterally marginate. Profemur broadened (FI 0.42). Metanotal groove essentially lacking (Figure 12); basal face of propodeum flattened, rounding into the shorter declivitous face $(\mathrm{BF} / \mathrm{DF} \approx 1.34)$. Petiole longer than high or wide (PLI 0.63, PWI ca. 0.57), laterally marginate, with a short anterior peduncle, prominent anteroventral tooth, and gradually rising anterodorsal face which rounds into a more steeply descending posterior face (Figure 12). In dorsal view petiole notably constricted anteriorly (PWI3 ca. 0.41), with concave posterodorsal margin. Postpetiole slightly broader than long (PPWI ca. 1.07). Integument apparently densely punctulate, opaque. Standing pilosity very sparse, present on pronotal humeri (1 pair), petiole (1 pair), postpetiole ( 2 pairs), and gaster; not seen 

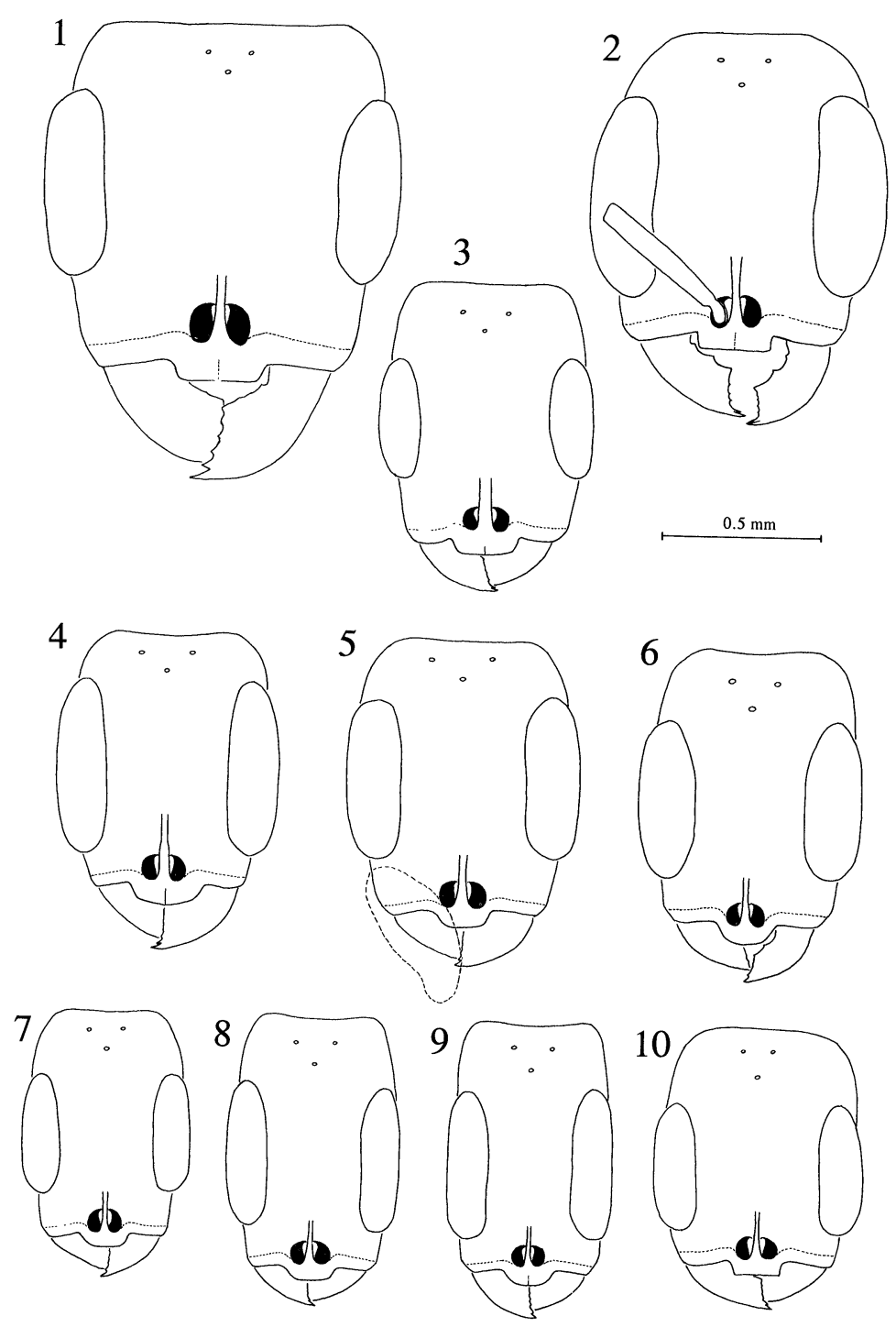
on the mesonotum, propodeum, and extensor faces of tibiae. Color apparently medium brown, antennae and clypeus lighter.

Comments: This species is considerably larger than all other Pseudomyrmex known from Dominican amber except $P$. avitus (q. v.). The head shape, mandibular dentition, marginate pronotum and petiole, and lack of metanotal groove are reminiscent of the extant Central American species P. rufomedius (F. Smith) and an undescribed Mexican species near $P$. rufomedius, but $P$. antiquus differs from these two in eye size, profemur shape, petiole shape, pilosity, and other details.

\section{Pseudomyrmex avitus, new species}

(Figure 11)

Holotype worker: Dominican amber (no further data) (\#PSWDA30) (UCBC).

Additional material: 1 male, Dominican amber, purchased by P. S. Ward from S. Hendrickson (no further data) (\#PSW-DA20) (MCZC).

Measurements, holotype worker: HW ca. 1.48, HL ca. 1.62, HD 1.08, EL 0.97, SL 0.88, FL 1.32, FW ca. 0.48, LHT 1.60, DPL 1.45, PL 0.68, PH 0.42, PPL ca. 0.63, DPW ca. 0.32, PPW ca. 0.50

Measurements, male: HW 1.48, HL 1.27, EL .75, SL ca. 0.20, LF1 0.15, LF2 0.36, FL 1.32, FW 0.31, LHT 1.67, PL 0.81, PH 0.32, DPW 0.36, MPW 0.19, PPL 0.74, PPW 0.67

Description, holotype worker: Large species with broad head and large, protruding eyes (CI ca. 0.91, REL ca. 0.60). (Head and mesosoma appear distorted in shape (bilaterally asymmetrical) in

Figures 1-10. Dominican amber Pseudomyrmex: frontal views of worker head. 1. P. antiquus, 2. P. baros, 3. P. coruscus, 4. P. macrops, 5. P. nexilis, 6. P. thecolor, 7. P. oryctus, 8 . P. prioris, 9. P. succinus, 10 . $P$. simplex. All drawings are to the same scale (scale line $=0.5 \mathrm{~mm}$ ) and all are of holotypes except Fig. 4 (paratype) and Fig. 10. Where obstructions of view occurred (of which a large one is indicated by dashed lines-see Figure 5) reconstruction was based on the assumption of bilateral symmetry or on extrapolation from oblique views. 


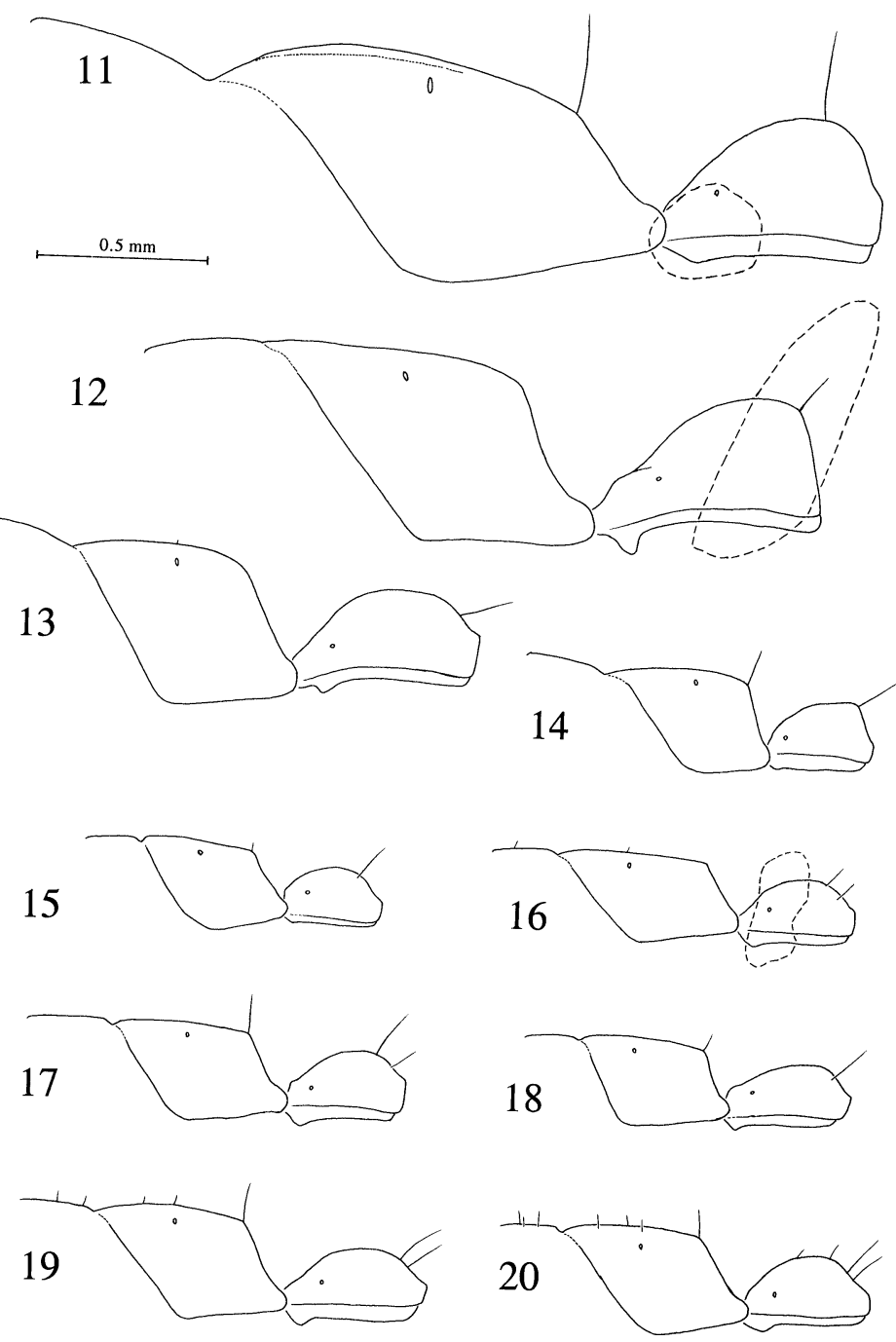


dorsal view through the amber, so head measurements and indices are approximate.) Masticatory margin of mandible with a strong apical tooth preceded by a subapical tooth and 7-8 small (worn) denticles. Median clypeal lobe tectiform, broadly rounded laterally. Frontal carinae subcontiguous $(\mathrm{FCI} \approx 0.02)$ and fusing with antennal sclerites anterolaterally. Second and third funicular segments almost twice as long as wide. Profemur relatively slender (FI ca. 0.36). Pronotum and anterior half of propodeum laterally marginate. Mesonotum sloping downwards towards basal face of propodeum, so that metanotal groove is deeply impressed in lateral view (Figure 11); basal face of propodeum convex in profile, rounding insensibly into the much shorter declivitous face (BF/DF $\approx 1.91$ ). Petiole short, high (PLI 0.62, PWI 0.48), laterally marginate, and without an anterior peduncle (PWI3 0.46) (Figure 11). Postpetiole longer than broad. Head and mesosoma finely coriarious-punctulate, opaque. Standing pilosity consisting of sparse but conspicuous long black hairs, distributed as follows: antennal scapes (2-3), upper half of head (3 long supraocular pairs and several shorter hairs), pronotum (2 pairs), propodeum (1 pair, at juncture of basal and declivitous faces), petiole (1 pair), postpetiole ( 2 pairs), gaster (including 12-14 hairs on abdominal tergite IV) and coxae; absent from extensor faces of tibiae. Dark brown to black; pronotum, mesonotum and protibia a contrasting pale luteous.

Description, male: Large, with broad head (CI 1.17) and moderately large eyes (REL 0.59) which protrude from the sides of the head. Mandibles with about 15 denticles on the masticatory margin proximal to the apical tooth; basal margin of mandibles edentate. Palp formula 6,4. SL $<$ LF2. (Funicular segments beyond LF2 are missing from the specimen.) Ocelli conspicuous, maximum diameters about $0.15 \mathrm{~mm}$. Posterior margin of head weakly concave in frontal view. Profemur very slender (FI 0.23). Mesonotum of moderate size and typical shape, neither constricted medially nor protruding anteriorly. Basal face of propodeum rounding broadly into the declivitous face. Petiole elongate (PLI 0.40, PWI 0.44, PWI3

Figures 11-20. Dominican amber Pseudomyrmex: left lateral views of worker propodeum and petiole. 11. P. avitus, 12. P. antiquus, 13. P. baros, 14. P. macrops, 15. P. oryctus, 16. $P$. prioris, 17. P. nexilis, 18. P. succinus, 19. P. thecolor, 20. $P$. vicinus. All drawings are of holotypes; other conventions as in Figures 1-10. 
0.53 ) without a distinctly constricted anterior peduncle, either in lateral or dorsal views; spiracles not protruding laterally. Petiole with a small anteroventral tooth. Postpetiole slender (PPWI 0.91). Pygidium simple in form, directed caudad (not recurved anteroventrally), its posterior margin weakly emarginate. Cerci long and slender. Hypopygium (abdominal sternite IX) difficult to see but apparently with a blunt triangular posterior margin. In lateral view distal end of paramere steeply wedge-shaped; preceding this is an apparent thin posterodorsal crest. In dorsal view distal end of paramere bluntly triangular and preceded by a mesial dorsoventral lobe. Head and mesosoma finely coriarious-punctulate, opaque. Standing pilosity rather sparse, present as black hairs on head (including 2 conspicuous supraocular pairs, similar in size and position to those of the worker), mesonotum, mesoscutellum, petiole, postpetiole, and gaster, not seen on extensor faces of tibiae, and almost absent (one short seta only) from propodeum. Appressed pubescence common on petiole, postpetiole, and gastric tergites. Apparently dark brown-black in color, with lighter lateral mottling on the mesonotum.

Comments: The large size ( $\mathrm{HW} \approx 1.48$ ), distinctive pilosity, deep metanotal impression (Figure 11), and anteriorly marginate propodeum easily distinguish this species from all others including $P$. antiquus. The association of the male with the $P$. avitus worker, although tentative, is supported by similarities in overall size (compare HW, LHT), mandibular dentition, eye size, petiole shape, pilosity, sculpture, and proportions of the head and profemur. Some features of $P$. avitus are shared with extant species in the $P$. gracilis group (9-10 teeth on the masticatory margin of worker mandible, marginate mesosoma, shape of the distal end of the paramere), the $P$. tenuis group (tectiform clypeus, sparse pilosity) or both (large size, elongate eyes, marginate pronotum, palp formula of 6,4). However, P. avitus differs from the former group by its apedunculate petiole and relative lack of pilosity, and from all $P$. tenuis group species by its mandibular dentition, deeply impressed metanotal groove, shape of the propodeum and specific distribution of standing pilosity. 


\section{Pseudomyrmex baros, new species}

(Figures 2, 13)

Holotype worker: Dominican amber, purchased by P. S. Ward (no further data) (\#PSW-DA18) (MCZC)

Paratype worker: Dominican amber, purchased for MCZC (no further data) (\#PSW-DA7) (MCZC)

Holotype measurements: HW 0.96, HL 1.01, EL 0.64, MFC ca. 0.02, SL 0.44, FL ca. 0.70, FW ca. 0.28, LHT 0.74, DPL 0.81, PL 0.55, PH 0.28, PPL ca. 0.42, DPW 0.23, MPW 0.13, PPW ca. 0.40 .

Paratype measurements: HW ca. 0.98, HL ca. 1.11, HD 0.63, EL 0.70, MFC ca. 0.02, SL 0.45, FL ca. 0.73 , FW ca. 0.26, LHT 0.75, DPL 0.81, PL 0.56, PH 0.28, PPL ca. 0.41, DPW 0.24, PPW 0.43 .

Description: Relatively large species (see measurements) with broad head (CI ca. 0.88-0.95) (in frontal view the head of the paratype worker appears more elongate than this because of a concave cut in the amber). Masticatory margin of mandibles with 6-7 teeth: a large apical tooth, a slightly smaller subapical tooth, 3-4 denticles, and a weakly acute (almost right-angled) apicobasal tooth; basal margin with a well developed mesial tooth, situated about midway between the proximal tooth and apicobasal tooth. Holotype: 4 labial palp segments, number of maxillary palp segments indeterminate; paratype: labial palps not visible but at least three maxillary palps discernable in lateral view, the terminal segment ca. $0.13 \mathrm{~mm}$ in length and almost twice as long as the penultimate segment, suggesting some fusion. Median clypeal lobe tectiform, laterally subangulate (Figure 2). Frontal carinae rather close (FCI 0.02), fusing anteriorly with the antennal sclerites. Second and third funicular segments slightly longer than broad. (In the holotype the left funiculus has the expected eleven segments, the right eight only-presumably an aberration.) Eyes large (REL 0.63-0.64). Profemur relatively slender (FI ca. 0.36-0.40). Pronotum laterally submarginate. In lateral view a broad impression formed at the juncture of the somewhat steeply descending posterior end of the mesonotum and the shallowly depressed anterior 
end of the propodeum, so that MPI $\approx 0.05$ although metanotal groove not narrowly incised (Figure 13). Basal face of propodeum convex, slightly longer than, and rounding gently into, the declivitous face. Petiole relatively long, low (PLI 0.50-0.51, PWI $0.42-0.43$, PWI3 0.13), apendunculate, dorsolaterally submarginate, and with an anteroventral tooth. Postpetiole about as long as wide (PPWI ca. 0.98-1.05). Head and mesosoma apparently densely punctulate and opaque. Standing pilosity limited in distribution: present on dorsum of head ( 3 pairs on the vertex-one pair posterior to the compound eye, two pairs near the ocelli), pronotum (2-3 pairs), propodeum (one or two short hairs on the basal face), petiole (one long pair), postpetiole ( 2 pairs), and gaster; absent from mesonotum and from extensor faces of tibiae. Paratype additionally with a pair of curved setae, ca. $0.10 \mathrm{~mm}$ in length, at the juncture of the basal and declivitous faces of the propodeum. Abdominal tergite IV with a mat of appressed pubescence. Color apparently medium-dark brown, postpetiole and base of gaster a little lighter.

Comments: P. baros is distinguishable from all other species described here by its size and mesosomal profile. The holotype and paratype workers agree reasonably well, although the paratype possesses an additional pair of erect setae on the propodeum. $P$. baros appears to be related to the extant Hispaniolan species $P$. haytianus (Wheeler \& Mann) on the basis of the following shared similarities: masticatory margin of the mandible with 6-7 teeth; mesial tooth on basal margin of mandible positioned at midlength; median clypeal lobe laterally angulate; head broad (CI > 0.85); size moderately large $(\mathrm{HW} \approx 1.00)$; profemur slender; basal face of propodeum convex and rounding broadly into declivitous face; petiole more or less apendunculate, dorsolaterally submarginate; and standing pilosity sparse on the mesosoma and tibiae. At the same time $P$. haytianus differs from $P$. baros in several features including its smaller eyes (REL $\approx 0.54$ ), loss of one labial palp segment, shallower metanotal impression and shorter pilosity. 


\section{Pseudomyrmex coruscus, new species}

(Figure 3)

Holotype worker: Dominican amber from Palo Quemado, Santiago Province purchased for MCZC from Geological Enterprises (\#PSW-DA13) (MCZC)

Measurements: HW 0.68, HL 0.87, EL 0.40, MFC ca. 0.04, SL ca. 0.32 , FL ca. 0.52 , FW 0.23, LHT ca. 0.54 , PL ca. 0.34 , PPL ca. 0.32 , DPW 0.24, MPW ca. 0.15, PPW 0.41.

Description: Head longer than broad (CI 0.78). Median clypeal lobe broadly rounded laterally. Frontal carinae not contiguous, separated by about basal scape width (FCI 0.06). Second and third funicular segments broader than long. Eyes relatively short (REL 0.46). Profemur moderately swollen (FI ca. 0.45). Metanotal groove well impressed. Basal face of propodeum subequal to, or slightly shorter than, declivitous face and rounding very broadly into it. Petiole relatively short and wide (PWI ca. 0.70), broad anteriorly (PWI3 ca. 0.62). Postpetiole broader than long (PPWI ca. 1.29). Head finely punctulate; punctulae separated by one to several times their diameters, interspaces smooth and shining. Dorsum of mesosoma, petiole and postpetiole similarly smooth and shiny, punctulae less evident. Standing pilosity not abundant: present on head ( 1 pair on the vertex), pronotum (2-3 pairs), petiole (1 pair), postpetiole ( 2 pairs), and gaster. Appressed pubescence apparently not dense on abdominal tergite IV, so that the latter has a strong sheen like most of the body. Color appearing to be dark brown to black.

Comments: The body sculpture and broad petiole and postpetiole distinguish this species from others described here. Also characteristic are the laterally rounded clypeal lobe, well separated frontal carinae, short eyes, form of propodeum, and sparse pilosity. 


\section{Pseudomyrmex macrops, new species}

(Figures 4, 14)

Holotype worker: Dominican amber, purchased for MCZC (no further data) (\#PSW-DA11) (MCZC).

Paratype worker: Dominican amber, purchased for MCZC (no further data) (\#PSW-DA10) (MCZC)

Additional material: 1 worker, Dominican amber from Santiago region (\#PSW-DA22) (from a series of six pieces of amber labeled H-10-114, UCBC). 1 worker, Dominican amber (no further data) (\#PSW-DA29) (UCBC).

Measurements, holotype: HW 0.65, HL 0.88, HD 0.45, EL 0.57, SL 0.29, FW 0.26, FL 0.53, LHT 0.54, DPL 0.55, PL 0.30, PH 0.20 , PPL ca. 0.24, DPW ca. 0.16, PPW ca. 0.32.

Measurements, paratype: HW 0.69, HL 0.90, HD ca. 0.48, EL 0.54, MFC ca. 0.02, SL 0.29, FW 0.24, FL 0.54, LHT 0.52, DPL ca. 0.55 , PL ca. $0.25, \mathrm{PH}$ ca. 0.20 , PPL ca. 0.24, DPW 0.18, PPW ca. 0.35.

Description: Masticatory margin of mandibles with 7 teeth (nontypes). Palp formula apparently 6,4 (definitely so in worker \#PSWDA29). Head longer than broad (CI 0.73-0.76, LCI 0.51-0.53). Median clypeal lobe strongly tectiform, rounded laterally. Frontal carinae subcontiguous (FCI ca. 0.03), fusing with the antennal sclerites anteriorly. Eyes very elongate (REL 0.60-0.65). Second and third funicular segments wider than long. Profemur broadened (FI 0.45-0.48). In lateral view mesonotum sloping posteriorly and meeting the propodeal dorsum at an angle (of about $140^{\circ}$ ) so that the metanotal groove is well marked (i.e., MPI ca. 0.07) but not impressed much below the level of the propodeal dorsum (Figure 14). Basal face of propodeum longer than declivitous face, mostly flat but somewhat depressed posteriorly before meeting the declivitous face at an angle. Petiole relatively short and high (PLI 0.67-0.81, PWI ca. 0.52-0.73), dorsolaterally submarginate, and with a gently sloping anterodorsal face which rounds sharply into a steep posterior face. Postpetiole considerably wider than long (PPWI ca. 1.34-1.46) and with a small but distinct anteroventral 
process. Head and mesosoma densely punctulate-coriarious and apparently opaque. Standing pilosity sparse, present on dorsum of head, pronotum (1 pair), juncture of basal and declivitous faces of propodeum (1 pair, not seen in paratype), petiole (1 pair), postpetiole (1-2 pairs), and gaster. Color appearing medium brown, the gaster perhaps a little darker.

Comments: Diagnostic features of $P$. macrops are the elongate eyes $(\mathrm{REL} \approx 0.62)$, tectiform clypeus, short high petiole with steep posterior face, broad postpetiole, mesosomal profile (see description above), and relatively small size $(\mathrm{HW}<0.75$, LHT $<0.60)$. The first four traits are characteristic of the P. sericeus group, but the last two are not.

Worker \#PSW-DA29 agrees with most parts of the detailed description above but it has a longer head (HL 0.98, CI 0.70, LCI 0.49), less broad postpetiole (PPWI ca. 1.22) and appears to be dark brown-black in color. Worker \#PSW-DA22 differs in minor ways from the type specimens: profemur broader (FI 0.51), petiole less angulate, and more standing pilosity visible on abdominal tergite IV. Although either of these might represent different species it seems prudent to include them under P. macrops until more material is available for study.

\section{Pseudomyrmex nexilis complex}

The six species described below share the following traits: relatively small size $(\mathrm{HW}<0.75$, LHT $<0.60)$; broadly rounded median clypeal lobe; subcontiguous and slightly elevated frontal carinae $(\mathrm{FCI} \approx 0.03$ ), fusing with the antennal sclerites anterolaterally; moderately elongate eyes (REL $0.52-0.57$ ); second and third funicular segments broader than long; broad profemur (FI > 0.44); basal and declivitous faces of propodeum meeting at an angle; and petiole apendunculate and relatively slender (PLI < 0.66), without a steep posterior face. These commonalities are not repeated in the individual descriptions below. Species differences are rather subtle but no more so than among closely related contemporary species of Pseudomyrmex. The members of the $P$. nexilis complex do not belong to any of the modern species groups of Pseudomyrmex but possess a mixture of some of the traits of the $P$. oculatus group 
(elongate head and eyes, fronto-clypeal structure) and the $P$. subtilissimus group (petiole shape and patterns of pilosity).

\section{Pseudomyrmex nexilis, new species}

(Figures 5, 17)

Holotype worker: Dominican amber, purchased for MCZC (no further data) (\#PSW-DA3) (MCZC)

Paratype workers: Two specimens from two pieces of Dominican amber, purchased for MCZC (no further data) (\#PSW-DA12, \#PSW-DA16) (MCZC)

Measurements, holotype: HW 0.72, HL 0.89, HD 0.52, EL 0.50, SL 0.27, FL 0.53, FW ca. 0.27, LHT 0.52, DPL 0.61, PL 0.35, PH 0.22, PPL ca. 0.30, DPW 0.20, PPW ca. 0.33.

Measurements, paratypes (\#PSW-DA12 and \#PSW-DA16, respectively): HW ca. $0.64,0.67$; HL $0.90,0.86$; HD 0.47, ca. 0.50; EL 0.49, 0.45; SL 0.29, 0.28; FL 0.52, 0.55; FW 0.24, 0.27; LHT 0.53, 0.53; DPL 0.61, ca. 0.58; PL ca. 0.35, 0.36; PH ca. $0.20,0.22$; PPL ca. 0.29 , ca. 0.32 ; DPW ca. $0.18,0.20$; PPW ca. 0.29 , ca. 0.35 .

Description: Masticatory margin of mandible with 6 teeth; basal margin with mesial tooth situated about midway between proximal tooth and apicobasal tooth; palp formula apparently 6,3 (paratype PSW-DA16). Head longer than broad (CI ca. 0.71-0.81, LCI $0.52-0.58$ ). Eyes elongate (REL 0.52-0.56). Profemur broad (FI 0.46 -ca. 0.51). Mesosoma flat in profile, with a shallowly impressed metanotal groove (MPI $\approx 0.03$ ). Basal face of propodeum about 1.5 times the length of the declivitous face. Petiole relatively short for the $P$. nexilis complex (PLI ca. 0.59-0.62, PWI ca. 0.52-0.56) (Figure 17). Postpetiole as broad or broader than long (PPWI ca. 1.00-1.10). Integument sculpture apparently punctulate to punctulate-coriarious. Standing pilosity sparse: present on dorsum of head, pronotal humeri (1 pair), propodeum at juncture of basal and declivitous faces ( 1 pair), petiole ( 2 pairs), postpetiole ( 2 pairs), and gaster. One or two additional, very short and inconspicuous hairs seen on mesonotum of holotype. Color medium brown 
(petiole, postpetiole and appendages appearing lighter brown in paratype PSW-DA16).

Comments: This is a relatively large species $(\mathrm{HW}>0.60)$ which can be separated from similar-sized species in the $P$. nexilis complex ( $P$. thecolor, $P$. vicinus) by the combination of broad head (CI $>0.68$ ) and short petiole (PLI > 0.55).

\section{Pseudomyrmex oryctus, new species}

(Figures 7, 15)

Holotype worker: Dominican amber, purchased for MCZC (no further data) (\#PSW-DA4) (MCZC).

Measurements: HW 0.52, HL 0.74, HD 0.41, EL 0.39, MFC ca. 0.02, SL 0.22, FL 0.41, FW 0.21, LHT 0.40, DPL ca. 0.48, PL 0.28 , PH 0.18, PPL ca. 0.24 , DPW ca. 0.18 , PPW ca. 0.28 .

Description: Small species (see measurements, especially HL and LHT). Palp formula 6,3 (right labial palp appearing 4p3, i.e., with partial fusion of third and fourth segments). Head longer than broad (CI 0.70) but not particularly elongate in lateral view (LCI 0.55). Eye length about one-half head length (REL 0.52). Profemur strongly swollen (FI 0.51). Dorsum of mesosoma rather flat in profile, metanotal groove weakly impressed (MPI ca. 0.02). Basal face of propodeum longer than declivitous face $(0.33 \mathrm{~mm}$ versus 0.21 $\mathrm{mm}$ ). Petiole rather short (PLI 0.63, PWI ca. 0.66). Postpetiole broader than long (PPWI ca. 1.20). Body (especially head and gaster) apparently smooth and shining, without any evident microsculpture. Standing pilosity sparse, present on head (including at least two very short pairs on the vertex), pronotum (1 pair), petiole (1 pair), postpetiole (1 pair), and gaster (absent from abdominal tergite IV except the posterior margin). Also a pair of short inconspicuous setae (ca. $0.025 \mathrm{~mm}$ long) at the juncture of the basal and declivitous faces of the propodeum. Color appearing medium brown, head a little darker than the mesosoma; malar area, petiole, postpetiole, and lateral parts of pronotum a lighter mottled yellow-brown; gaster dark brown. 
Comments: There are three small species in the $P$. nexilis complex $(\mathrm{HW}<0.57, \mathrm{FL}<0.50) . P$. oryctus is distinguished from the other two ( $P$. prioris, $P$. succinus) by its relatively broad head, short petiole, and robust propodeum.

\section{Pseudomyrmex prioris, new species}

(Figures 8, 16)

Holotype worker: Dominican amber, from "Valla Guana" (R. Harrison) (\#PSW-DA15) (MCZC)

Measurements: HW 0.53, HL 0.86, HD 0.47, EL 0.47, MFC ca. 0.01 , SL 0.22, FL 0.47, FW 0.23, LHT 0.47, DPL 0.60, PL 0.35, PH 0.21, PPL ca. 0.30, DPW ca. 0.19, PPL ca. 0.33 .

Description: Masticatory margin of mandibles with 6 teeth. Palp formula apparently 6,4. Head considerably longer than broad (CI 0.62 ), with concave posterior margin, but not particularly elongate in lateral view (LCI 0.55). Scapes short (SL/HL 0.26). Eyes elongate (REL 0.54 ). Profemur notably broadened (FI 0.49). Mesosomal dorsum rather flat in profile, metanotal groove very shallowly impressed (MPI 0.02). Basal face of propodeum almost flat, and notably longer (ca. $0.43 \mathrm{~mm}$ ) than the declivitous face (ca. 0.23 $\mathrm{mm}$ ). Petiole relatively short and high (PLI 0.59, PWI ca. 0.54) (Figure 16) compared to that of $P$. succinus. Head and mesosoma densely punctulate-coriarious and apparently opaque, including the area immediately posterior to the compound eye. Standing pilosity short and inconspicuous, apparently absent from the posterior half of the dorsum of the head; very short hairs $(<0.04 \mathrm{~mm})$ seen on mesosoma dorsum as follows: $3-4$ on the pronotum, two on the mesonotum, and one on the basal face of the propodeum. Longer standing hairs $(0.05$ to $0.08 \mathrm{~mm})$ on the pronotal humeri (1 pair), petiole ( 2 pairs), postpetiole ( 2 pairs), and gaster; apparently absent from the juncture of the basal and declivitous faces of the propodeum (missing?). Appearing orange-brown in color, with a dark brown head and with paired anterolateral fuscous patches on abdominal tergite IV. 
Comments: Notable features of $P$. prioris are its small size and long basal face of propodeum. It differs from the otherwise similar $P$. succinus by its possession of a less dorsoventrally compressed head (compare LCI) and shorter petiole (PLI > 0.55).

\section{Pseudomyrmex succinus, new species}

(Figures 9, 18)

Holotype worker: Dominican amber from Palo Alto region, purchased by P. S. Ward from S. Hendrickson (\#PSW-DA17) (MCZC).

Paratype workers: Three workers in three separate pieces of Dominican amber, purchased for MCZC (no further data) (\#PSW-DA1, \#PSW-DA2, and \#PSW-DA9); two workers in two pieces of Dominican amber from Santiago region (\#PSWDA23, \#PSW-DA24) (from a series of six pieces of amber labeled H-10-114, UCBC).

Paratype queen: Dominican amber, purchased for MCZC (no further data) (\#PSW-DA5).

Additional material: one worker in Dominican amber from Santiago region (\#PSW-DA25) (from a series of six pieces of amber labeled H-10-114, UCBC).

Measurements, holotype: HW 0.51, HL 0.84, HD ca. 0.37, EL 0.47, SL 0.21, FL 0.47, FW ca. 0.22, LHT 0.43, DPL 0.49, BF 0.37 , DF 0.18 , PL 0.36, PH 0.19, DPW ca. 0.18, PPL 0.26, PPW ca. 0.29 .

Measurements, paratype workers ( $\mathrm{n}=5$ unless otherwise noted): HW 0.48-ca. $0.55(\mathrm{n}=4)$, HL 0.81-0.89, HD 0.36-0.42, EL $0.45-0.50$, SL $0.21-0.23$, FL ca. $0.40-0.49$, FW 0.21-0.24, LHT ca. $0.40-0.50$, DPL $0.50-0.57$, PL 0.33-0.37, PH ca. 0.16-0.20, DPW ca. $0.16-0.18(n=3)$, PPL ca. $0.29-0.33 \quad(n=4)$, PPW ca. $0.28-0.32(n=3)$.

Measurements, paratype queen: HW ca. 0.47, HL 0.95, HD 0.40, EL 0.50, SL 0.23, FL 0.44, FW 0.24, LHT 0.45, PL ca. 0.39, DPW ca. 0.19, PPL 0.35, PPW 0.35. 
Description (worker): Small species $(\mathrm{HW}<0.60$, LHT $<0.52$ ) with elongate head (CI ca. 0.59-0.63), especially in lateral view (LCI 0.41-0.47). Palp formula apparently 6(or 5),4. Scapes short (SL/HL 0.25-0.27). Eyes elongate (REL 0.55-0.57). Profemur broad (FI ca. 0.45-0.54). Mesosoma rather flat in profile, with a very shallow metanotal groove $(\mathrm{MPI} \approx 0.03)$. Basal face of propodeum twice as long as declivitous face or almost so (BF/DF ca. 1.79-2.07). Petiole elongate and low (PLI ca. 0.44-0.54, PWI ca. 0.45-0.55) (Figure 18). Postpetiole about as broad as long. Head and mesosoma apparently densely punctulate-coriarious and opaque; in the holotype and at least some paratypes the area immediately posterior to the compound eye has the punctulae less dense, separated by one to several diameters, with the interspaces smooth and shining. Standing pilosity scarce: present on pronotum (1 pair), at or near the juncture of the basal and declivitous faces of the propodeum (1 pair, not always discernable), on the petiole (1 pair), postpetiole (1-2 pairs), and gaster; also one or two very short $(<0.04 \mathrm{~mm}$ long), inconspicuous hairs on the pronotum, mesonotum and basal face of propodeum (these small hairs not seen in all specimens). Color appearing light to medium-dark brown, head and gaster dark brown.

The non-paratype worker (\#PSW-DA25) agrees with most aspects of the above description but it is rather small and slender (HW 0.48, LHT 0.40, CI 0.58, LCI 0.40, PLI 0.42) and short standing hairs are commoner on the head and the mesosoma.

Description (queen): Similar to the worker in size, fronto-clypeal structure, funicular proportions, petiole shape, sculpture, and pilosity. Profemur broad (FI 0.54) and head more elongate (CI ca. 0.50 ), as expected. Forewing with two closed cubital cells. Color dark brown, as in some of the paratype workers.

Comments: $P$. succinus is distinguished from other members of the $P$. nexilis complex by its elongate and dorsoventrally compressed head $(\mathrm{CI}<0.66, \mathrm{LCI}<0.50)$. Also characteristic are the small size $(\mathrm{HW}<0.60)$ and slender petiole $(\mathrm{PLI}<0.56)$. 


\section{Pseudomyrmex thecolor, new species}

(Figures 6, 19)

Holotype worker: Dominican amber, purchased in jewelry store in Santo Domingo by B. Cole (no further data) (\#PSW-DA19) (MCZC)

Additional material: 1 worker, Dominican amber, purchased for MCZC (no further data) (\#PSW-DA6) (MCZC); 1 worker, Dominican amber (no further data) (\#PSW-DA28) (UCBC).

Measurements, holotype: HW 0.69, HL 0.93, HD 0.50, EL ca. 0.52, SL 0.30, FL 0.57, FW 0.27, LHT 0.53, DPL 0.66, BF 0.43, DF 0.29, PL 0.41, PH 0.21, DPW ca. 0.20, PPL 0.31, PPW ca. 0.36 .

Description: Medium-sized species, with head longer than broad (CI 0.75) and apparently somewhat compressed in lateral view (LCI 0.54) but not as strongly as in P. succinus. Palp formula apparently 6,4. Eyes elongate (REL 0.56). Posterior margin of head weakly concave. Profemur swollen (FI 0.48). In lateral view metanotal groove shallowly but distinctly notched (MPI 0.04). Basal face of propodeum weakly convex, about 1.5 times longer than declivitous face (Figure 19). Petiole long, low, apendunculate (PLI 0.51, PWI ca. 0.49). Postpetiole broader than long (PPWI ca. 1.15). Body with punctulate-coriarious sculpture, apparently somewhat effaced, the intergument appearing sublucid. Standing pilosity more common and conspicuous than in most other Dominican amber Pseudomyrmex: present on upper third of head, pronotum (ca. 5 setae, including one long pair of ca. $0.14 \mathrm{~mm}$ length at the pronotal humeri), mesonotum (ca. 4 setae), basal face of propodeum (7-8 setae, including one long pair at the juncture of the basal and declivitous faces), petiole ( 2 pairs), postpetiole (2-3 pairs), and gaster (including 2-3 setae on abdominal tergite IV anterior to the row of hairs on the posterior margin). Color uniform dark brown.

The non-paratype workers (\#PSW-DA6, \#PSW-DA28) agree with the holotype in most respects (HW 0.67 (both); LHT ca. 0.53, 0.54; CI 0.75 (both); PLI 0.48 (both); PWI 0.56, ca. 0.46; PPWI 1.18 , ca. 0.97 ) but the standing pilosity is much less evident and 
the heads of both workers appear more compressed in lateral view (LCI 0.48, 0.45).

Comments: The combination of moderate size (HW >0.65, LHT $>$ 0.50 ), elongate petiole (PLI $<0.55$ ), and (in the holotype) relatively frequent standing pilosity distinguishes this species from others in the $P$. nexilis complex.

\section{Pseudomyrmex vicinus, new species}

(Figure 20)

Holotype worker: Dominican amber from Santiago region (\#PSWDA26) (from a series of six pieces of amber labeled H-10-114, UCBC).

Paratype worker: Dominican amber from Santiago region (\#PSWDA27) (in the same piece of amber as holotype, UCBC)

Additional material: One worker, Dominican amber, purchased for MCZC (no further data) (\#PSW-DA8) (MCZC).

Measurements, holotype: HW 0.61, HL 0.93, HD 0.49, EL 0.50, SL 0.24 , FL 0.51, FW 0.25, LHT 0.46, DPL 0.58, BF 0.40, DF 0.24 , PL 0.35, PH 0.22, DPW 0.19, PPL 0.29, PPW 0.37.

Measurements, paratype: HW 0.61, HL 0.96, HD 0.51, EL 0.51, SL 0.25, FL 0.52, FW 0.26, LHT 0.48, DPL ca. 0.61, PL 0.36, PH 0.23, DPW 0.20, PPL ca. 0.31, PPW 0.36.

Description: Head notably longer than broad (CI 0.63-0.66, LCI 0.53). Palp formula apparently 6,3. Scapes short (SL/HL 0.26). Eyes elongate (REL $0.53-0.54$ ). Profemur conspicuously broadened (FI 0.50-0.51). Metanotal groove lightly impressed (MPI $\approx$ 0.03 ). Petiole relatively short (PLI 0.62-0.63, PWI 0.55-0.57). Postpetiole broader than long (PPWI ca. 1.17-1.26). Head and mesosoma with punctulate-coriarious sculpture and opaque. Standing pilosity rather common: short (ca. $0.03 \mathrm{~mm}$ long) hairs present on head, mesosoma (ca. 15 visible in profile on mesosoma dorsum), petiole, postpetiole, and gaster; longer (ca. 0.08-0.12 mm) setae present on pronotal humeri (1 pair), at juncture of basal and declivitous faces of propodeum (1 pair), petiole ( 2 pairs), and postpetiole ( 2 pairs). Erect setae absent from the extensor faces of the 
tibiae. Fine mat of appressed pubescence present on gaster. Color apparently dark brown, petiole, postpetiole and appendages lighter.

Non-type worker (\#PSW-DA8) similar to the preceding (HW 0.58 , CI 0.62, LCI 0.51, SL/HL 0.27, REL 0.55, FI 0.49, PLI 0.60, PWI 0.52) but short, standing pilosity less evident.

Comments: $P$. vicinus is characterized by the elongate head, short scapes, relatively short petiole and conspicuous standing pilosity.

\section{Pseudomyrmex simplex (F. Smith)}

(Figure 10)

One worker: Dominican "amber" from Cotui, center of Dominican Republic, purchased for MCZC from R. Harrison (\#PSWDA14) (MCZC).

Measurements: HW 0.65, HL 0.78, EL 0.42, FW 0.21, FL 0.46, LHT 0.47, PL ca. 0.32 .

Description: Specimen largely obscured dorsally by a series of contiguous bubbles which mimic, in a distorted fashion, the body parts that they cover; but enough features visible from other angles to produce the following description. Small species, with moderately elongate eyes (REL 0.53) and somewhat broad head (CI $0.83)$. Masticatory margin of mandibles with about five teeth. Palp formula apparently 5,3. Median clypeal lobe laterally angulate. Frontal carinae closely contiguous, MFC much less than basal scape width. Profemur moderately swollen (FI 0.45). Petiole, as seen from an oblique ventral view, apparently similar in size and shape to that of $P$. simplex. Body shining, head apparently smooth with scattered punctulae. Standing pilosity scarce, evidence of setae on the pronotum, postpetiole, and gaster. Color appearing as pale yellow-brown or orange-brown.

Comments: The mandibular dentition, laterally angulate clypeal lobe, apparent palp formula of 5,3, and scarcity of standing pilosity place this specimen in the $P$. pallidus group. With respect to the characters that can be observed it is very similar to the extant species $P$. simplex (F. Smith), which is widespread throughout the 
Neotropics, although the head is rather broad and the profemur relatively slender for this species (see description in Ward, 1985). The pale Cotui "amber", in which the specimen is encased, is evidently younger than most Dominican amber including all other material examined in this study, and is perhaps more properly termed copal (Schlee, 1984; see also Wilson, 1988).

\section{Key to DOMINICAN AMBER PSEUDOMYRMEX BASED ON THE WORKER CASTE}

1(a) Medium to large species; HW $>0.90$, FL $>0.65$, LHT $>0.70$

1(b) Smaller species; HW $<0.80$, FL $<0.60$, LHT $<0.65 \ldots \ldots \ldots . . .3$

2 (a) Very large species (HW $\approx 1.48, \mathrm{LHT} \approx 1.60)$; eyes elongate $(\mathrm{REL} \approx 0.60)$; metanotal groove strongly impressed in lateral view (Figure 11) avitus

2 (b) Large species $(\mathrm{HW} \approx 1.20$, LHT $\approx 0.97)$; eyes moderately elongate $(\mathrm{REL} \approx 0.52)$; metanotal impression lacking (Figure 12) (see also 2(c)) antiquus

2 (c) Medium-sized species $(\mathrm{HW} \approx 0.97$, LHT $\approx 0.75$ ); eyes very elongate (REL $0.63-0.64$ ); metanotal groove moderately impressed (Figure 13) baros

3(a) Median clypeal lobe laterally angulate; frontal carinae closely contiguous (MFC $<0.02$ and much less than basal scape width); eyes moderately elongate (REL ca. 0.52); head appearing rather broad in lateral view (LCI >0.60); an extant species known as a fossil only from Cotui copal simplex (F. Smith)

3(b) Median clypeal lobe rounded laterally; other characters variable; fossils from older amber

4(a) Eyes short, less than one half head length (REL 0.46); frontal carinae separated by about basal scape width $(\mathrm{FCI} \approx 0.06)$ (Figure 3); medium-sized species (HW 0.68, LHT ca. 0.54) with shiny integument coruscus

4(b) Eyes more elongate (REL $>0.50$ ); frontal carinae more closely contiguous $(\mathrm{FCI}<0.04)$ (e.g., Figures $4-9$ ); body sculpture usually predominantly punctulate-coriarious and opaque 
5(a) Eyes very elongate (REL $0.60-0.65$ ); petiole short, high (PLI $0.67-0.81$ ) with a steep posterior face (Figure 14); postpetiole very broad (PPWI ca. 1.22-1.46) macrops

5(b) Eyes less elongate (REL 0.52-0.57); petiole longer, lower (PLI 0.42-0.63) with a more gently inclined posterior face (e.g., Figures 15-20); postpetiole usually less broad (PPWI < 1.30)

6(a) Small (HW 0.52, HL 0.74, LHT 0.40) but robust species with relatively broad head $(C I 0.70)$, short eyes $(E L \approx 0.39$, REL 0.52 ), short basal face of propodeum (BF/DF 1.57), and short petiole (PLI 0.63) (Figure 15) oryctus

6(b) $\mathrm{HL}>0.78$, EL $>0.42$; larger in other dimensions (HW $>$ 0.62 , LHT $>0.50)$ and/or more slender $(\mathrm{CI}<0.68$, PLI $<$ $0.56)$

7(a) Larger species (HW 0.64-0.72, FL 0.52-0.57, LHT $0.52-0.54)$ with broader head (CI $0.71-0.81$ ) and longer scapes (SL/HL 0.31-0.32, SI2 0.54-0.62)

7(b) Smaller species (HW $<0.63$, FL $<0.53$, LHT $<0.52)$ with more elongate head (CI 0.58-0.66) and shorter scapes (SL/HL 0.25-0.27, SI2 0.44-0.49)

8(a) Petiole slender, about twice as long as high (PLI 0.48-0.51); basal face of propodeum weakly convex; standing pilosity evident on the mesosoma dorsum (at least in the holotype) (Figure 19) thecolor

8(b) Petiole shorter (PLI 0.59-0.62); basal face of propodeum flatter and standing pilosity sparser (Figure 17) nexilis

9(a) Petiole slender (PLI 0.42-0.54) (Figure 18); head elongate and strongly dorsoventrally compressed (CI $0.58-0.63$, LCI 0.40-0.47); size small (HW 0.48-0.55, FL 0.40-0.49, DPL 0.49-0.57) succinus

9(b) Petiole shorter (PLI 0.59-0.63) (Figures 16, 20); head somewhat broader and less compressed in lateral view (CI $0.62-0.66$, LCI $0.51-0.55$ ); DPL $0.58-0.66$, averaging larger in the other dimensions

10(a) Larger species (HW 0.58-0.61, FL 0.51-0.52) with head more strongly compressed dorsoventrally (LCI 0.51-0.53); standing pilosity conspicuous on mesosoma dorsum (may be missing or difficult to see) (Figure 20) vicinus 
10(b) Small species (HW 0.53, FL 0.47) with head less dorsoventrally compressed (LCI 0.55 ); standing pilosity less conspicuous on mesosoma dorsum (Figure 16) prioris

\section{The Contemporary PSeudomyrmex Fauna of the Antilles}

Of the 33 extant species of Pseudomyrmex found in the West Indies, 20 are confined, within this region, to Trinidad or adjacent islands of the Lesser Antilles (Table 1). There are no endemics among these; all are widespread South American species, some of which also occur in Central America. Only five of these 20 species have dispersed northward beyond Trinidad. Four species of Pseudomyrmex are present in both the Greater and Lesser Antilles. All of these are found throughout the Neotropics. This leaves nine species whose West Indian distributions encompass only the Greater Antilles (see upper third of Table 1). This group comprises three endemics (two from Cuba, one from Hispaniola), four species whose distributions are primarily northern Neotropical, one primarily South American species, and one pan-Neotropical species or species complex.

This pattern of distributions among the contemporary Pseudomyrmex of the West Indies suggests a predominantly youthful, dispersive fauna, a description which may apply to most West Indian ant genera (Wilson, 1988). This is also supported by a consideration of taxonomic relationships. Within the genus Pseudomyrmex as a whole I recognize nine major species groups (Ward, 1989) which contain about $85 \%$ of the estimated $150-200$ species; a miscellaneous selection of taxonomically isolated species (incertae sedis) makes up the remaining $15 \%$. In conformity with the pattern seen among mainland species most of the present-day West Indian species (27 out of 33) fall into the major species groups. Even four of the six taxonomically isolated species are widespread on the mainland, although they are not part of species-rich clades. Thus one does not get the impression that the West Indies are a haven for relicts in the genus Pseudomyrmex. One exception is provided by the endemic Hispaniolan species, $P$. haytianus. This species is not a member of any of the major species groups (Kempf's (1961) placement of it in the $P$. pallens group is not supported by a consideration of fronto-clypeal structure, palp formula, or male 
genitalia). $P$. haytianus is also one of the few extant species of Pseudomyrmex which shows any obvious affinities with the Dominican amber fauna (see below).

The other two Antillean endemics are confined to Cuba. $P$. pazosi (Santschi) is a member of the $P$. pallidus group and a close relative of the pan-Neotropical species, $P$. simplex. The identity of $P$. opacior (Forel) is unclear: the type is lost and no additional material is known. From the original description I would guess that it might represent a species similar to $P$. viduus (F. Smith) but this remains speculation until $P$. opacior is rediscovered.

\section{DISCUSSION}

1) Relationships of the Dominican amber Pseudomyrmex to extant taxa

All of the Dominican amber pseudomyrmecines which I have examined possess the characteristic features of the genus Pseudomyrmex (for diagnosis see Ward, 1990). As such they are clearly referable to this genus rather than one of the other two pseudomyrmecine genera, known from Amazonia and the Old World tropics, respectively. However none of the eleven fossil species of Pseudomyrmex fits comfortably within any of the species groups to which most extant congeners belong. (In contrast the specimen from Cotui copal corresponds to a modern species in the $P$. pallidus group.) Some of the species (e.g. those of the $P$. nexilis complex) possess a mixture of traits, characteristic of two or more species groups; others exhibit some but not all of the features of a particular group (e.g. $P$. macrops in relation to the $P$. sericeus group); still others show a combination of traits not seen in present-day taxa. Thus the Dominican amber offers a tantalizing glimpse of an earlier Pseudomyrmex radiation now largely eclipsed by modern forms.

One of the fossil species, $P$. baros, appears to be related to the endemic Hispaniolan species, $P$. haytianus, on the basis of several shared similarities (see above under $P$. baros) of which at least some are likely to be derived (e.g. clypeal structure, mesosomal shape). $P$. haytianus, in turn, bears some resemblance in worker morphology and male genitalia to two undescribed species of Pseudomyrmex known from mostly upland sites in Mexico and 


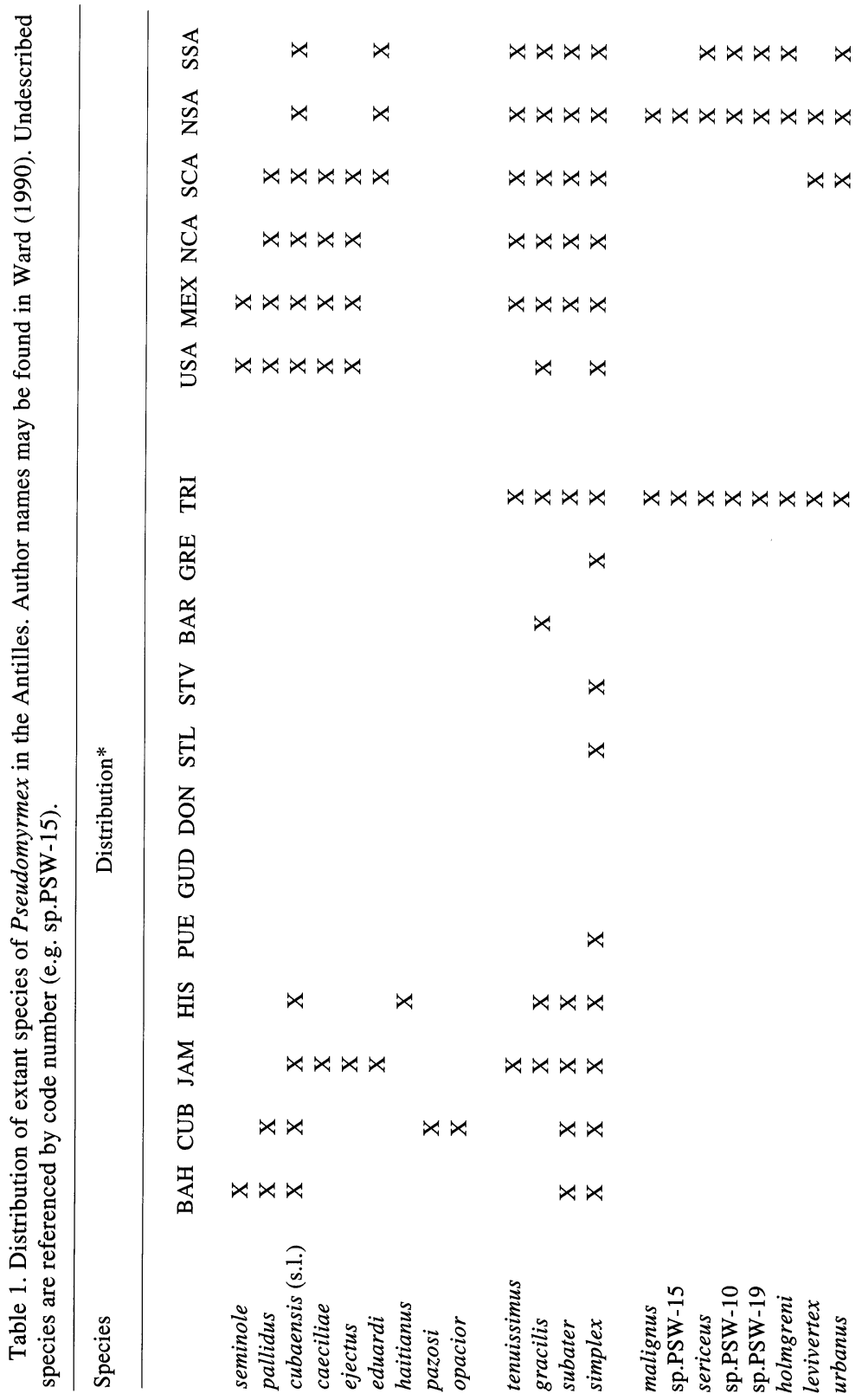




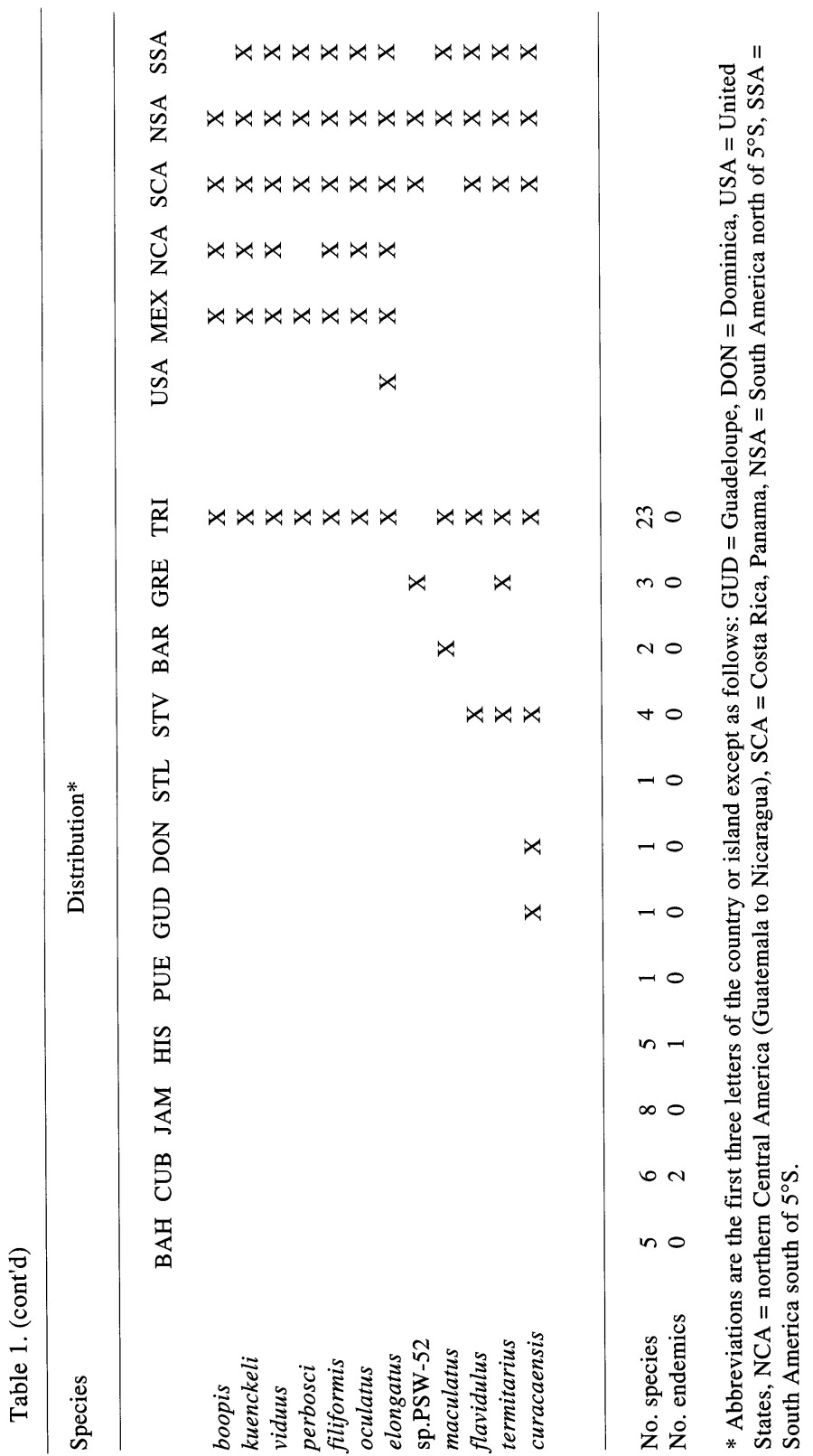


Central America. Another fossil species, $P$. antiquus, shows evidence of affinity to $P$. rufomedius and a related undescribed species, both taxonomic oddities known only from Mexico and Guatemala. These data, together with Wilson's (1985b) finding of a Dominican amber army ant, Neivamyrmex ectopus, with apparent relatives in northern Central America, suggest an historical link between the ant faunas of the Greater Antilles and mainland Mesoamerica, a biogeographic pattern seen in other Caribbean insects (Liebherr, 1988). Such distribution patterns and relationships support the assertion that during the middle Tertiary Hispaniola was part of, or adjacent to, a larger (continental) land mass (Wilson, 1988).

\section{2) Faunal turnover and decline}

The relatively small amount of material available for study reveals nevertheless a remarkably species-rich Pseudomyrmex fauna in Dominican amber: eleven species among 29 individuals (excluding the Cotui specimen in copal) compared with five species on Hispaniola today (see above and Table 1). This points to a $50 \%$ loss in species diversity since the Oligocene/Miocene. This is probably a conservative estimate. Among the five contemporary species, only one ( $P$. haytianus) appears to be widespread, based on the amount of material in collections. Thus one could expect a random sample of twenty-nine resin-trapped Pseudomyrmex specimens from the Dominican Republic today to typically include no more than two or three species, perhaps only one (this assertion can be field-tested). Note that the eleven fossil species are represented by the following numbers of individuals each $(\mathrm{w}=$ worker, $q=$ queen, $\mathrm{m}=$ male): $7 \mathrm{w}+1 \mathrm{q}, 4 \mathrm{w}, 3 \mathrm{w}, 3 \mathrm{w}, 3 \mathrm{w}, 2 \mathrm{w}, 1 \mathrm{w}+$ $1 \mathrm{~m}, 1 \mathrm{w}, 1 \mathrm{w}, 1 \mathrm{w}, 1 \mathrm{w}$. This suggests a diverse fauna and the likelihood that additional fossil taxa remain to be discovered. Hence the reduction in species richness could well be closer to an order of magnitude. Nevertheless it should be acknowledged that the Dominican amber samples are probably not all of equal age (even leaving aside the Cotui specimen) and this will tend to inflate the number of fossil species.

The existence of a depauperate Pseudomyrmex fauna on Hispaniola today is paralleled by a low diversity of other arboreal ants. 
Indeed entire genera and species groups such as Azteca, Dolichoderus (including Dolichoderus s.s., Hypoclinea, and Monacis), Leptothorax (Nesomyrmex), and Paraponera have gone extinct on the island since the Oligocene/Miocene (Wilson, 1985d) and almost no new genera have replaced them in the arboreal niche (Myrmelachista is one exception). Thus the stability of the Hispaniolan ant fauna, as seen by a comparable number of fossil and extant genera or subgenera (Wilson, 1985d), is rather deceptive. Most of the apparent new arrivals are ground-dwelling taxa, including in the very recent past a few tramp species of Old World origin. For ants nesting or foraging in arboreal habitats (arguably the group best represented in fossil amber) the genus-level comparisons of Wilson (1985d) already suggest a decline in diversity, and this is reinforced by the findings presented here on Pseudomyrmex.

The factors responsible for this change are not known, but the loss of arboreal species suggests that there has been a reduction or degradation of mesic forest habitats on Hispaniola within the last 20-40 million years, perhaps due to increasing insularization together with periods of cooler or drier climate. Study of material in the much younger Cotui amber/copal might help to resolve the question of when such changes occurred.

\section{CONCLUSIONS}

The present-day Pseudomyrmex fauna of the West Indies presents a youthful aspect. Most of the 13 species found in the Greater Antilles (of which 5 occur in Hispaniola) are also widespread in Central and/or South America, and they belong to species groups whose diversity is greatest in equatorial regions of South America. Some of these species groups may well have arrived in Central America and the Greater Antilles relatively recently, at about the time of formation of the Panamanian land bridge in the late Miocene or Pliocene. A rather different Pseudomyrmex assemblage is seen in the Dominican amber of Oligocene/early Miocene age. The eleven species, newly described here from 28 pieces of amber, cannot be placed in any of the modern species groups. (In contrast, a single Pseudomyrmex specimen examined in Cotui amber/copal belongs to a living species, present in the Antilles today.) Indeed the taxonomic affinities of most of the Dominican amber 
Pseudomyrmex remain obscure, suggesting that they are part of now extinct lineages. However one fossil taxon, $P$. baros, appears to be related to $P$. haytianus, an extant and taxonomically isolated species endemic to Hispaniola, with distant relatives in upland localities of Central America; and another fossil species, $P$. antiquus, shows evidence of affinities to two relictual species of Pseudomyrmex found in Mexico and Guatemala. Thus the Dominican amber Pseudomyrmex appear to be part of an early Mesoamerican/Antillean radiation of the genus, remnants of which persist today much reduced by extinction and largely replaced by more modern forms. On Hispaniola, in contrast to the mainland, replacement has lagged behind extinction, with a resultant marked loss in species diversity.

\section{ACKNOWLEDGMENTS}

I am grateful to Ed Wilson who has been accumulating specimens of Dominican amber Pseudomyrmex in the MCZC for several years, and gently reminding me of their existence. Additional valuable material was received from Blaine Cole, George Poinar, and Susan Hendrickson. George Poinar kindly reshaped and polished several pieces of amber. This study was supported by NSF grant BSR 9006393.

\section{Literature Cited}

BARONI URBANi, C.

1980a. First description of fossil gardening ants. (Amber Collection Stuttgart and Natural History Museum Basel; Hymenoptera: Formicidae. I: Attini). Stuttg. Beitr. Naturk. (B)54:1-13.

1980b. Anochetus corayi n. sp., the first fossil Odontomachiti ant. (Amber Collection Stuttgart: Hymenoptera, Formicidae. II: Odontomachiti). Stuttg. Beitr. Naturk. (B)55: 1-6.

1980c. The first fossil species of the Australian ant genus Leptomyrmex in amber from the Dominican Republic. (Amber Collection Stuttgart: Hymenoptera, Formicidae. III: Leptomyrmicini). Stuttg. Beitr. Naturk. (B)62: 1-10.

1980d. The ant genus Gnamptogenys in Dominican Amber. (Amber Collection Stuttgart: Hymenoptera, Formicidae. IV: Ectatommini). Stuttg. Beitr. Naturk. (B)67: 1-10.

Baroni Urbani, C., SAUnders, J. B.

1982. The fauna of the Dominican Republic amber: The present status of knowledge. Trans. 9th Caribbean Geol. Conf., Santo Domingo, Dominican Republic, 1980, 1: 213-223. 
Baroni urbani, C., Wilson, E. O.

1987. The fossil members of the ant tribe Leptomyrmecini (Hymenoptera: Formicidae). Psyche 94: 1-8.

KEMPF, W. W.

1961. Estudos sôbre Pseudomyrmex. III. (Hymenoptera: Formicidae). Studia Entomol. 4: 369-408.

Krishna, K., Grimaldi, D.

1991. A new fossil species from Dominican amber of the living Australian termite genus Mastotermes (Isoptera: Mastotermitidae). Amer. Mus. Novitates 3021: 1-10.

Lambert, J. B., Frye, J. S., Poinar, G. O., JR.

1985. Amber from the Dominican Republic: an analysis by nuclear magnetic resonance spectroscopy. Archeometry 27: 43-51.

LIEBHERR, J. K. (ED.)

1988. Zoogeography of Caribbean insects. Ithaca: Cornell University Press, ix +285 pp.

SCHLEE, D.

1984. Notizen über einige Bernsteine und Kopale aus aller Welt. Pp. 29-62 in: Schlee, D. (ed.) Bernstein-Neuigkeiten, Stuttg. Beitr. Naturk. (C)18: 1-100.

WARD, P. S

1985. The Nearctic species of the genus Pseudomyrmex (Hymenoptera: Formicidae). Quaest. Entomol. 21: 209-246.

1989. Systematic studies on pseudomyrmecine ants: revision of the Pseudomyrmex oculatus and $P$. subtilissimus species groups, with taxonomic comments on other species. Quaest. Entomol. 25: 393-468.

1990. The ant subfamily Pseudomyrmecinae (Hymenoptera: Formicidae): generic revision and relationship to other formicids. Syst. Entomol. 15: 449-489.

1991. Phylogenetic analysis of pseudomyrmecine ants associated with domatia-bearing plants. Pp. 335-352 in: Huxley, C. R., Cutler, D. F. (eds.) Ant-plant interactions. Oxford: Oxford Univ. Press, xviii +601 pp.

WiLson, E. O.

1985a. Ants of the Dominican amber (Hymenoptera: Formicidae). 1. Two new myrmicine genera and an aberrant Pheidole. Psyche 92: 1-9.

1985b. Ants of the Dominican amber (Hymenoptera: Formicidae). 2. The first fossil army ants. Psyche 92: 11-16.

1985c. Ants of the Dominican amber (Hymenoptera: Formicidae). 3. The subfamily Dolichoderinae. Psyche 92: 17-37.

1985d. Invasion and extinction in the West Indian ant fauna: evidence from the Dominican amber. Science 229: 265-267.

1986. Ants of the Dominican amber (Hymenoptera: Formicidae). 4. A giant ponerine in the genus Paraponera. Isr. J. Entomol. 19: 197-200.

1988. The biogeography of the West Indian ants (Hymenoptera: Formicidae). Pp. 214-230 in: Liebherr, J. K. (ed.) Zoogeography of Caribbean insects. Ithaca: Cornell University Press, ix +285 pp. 

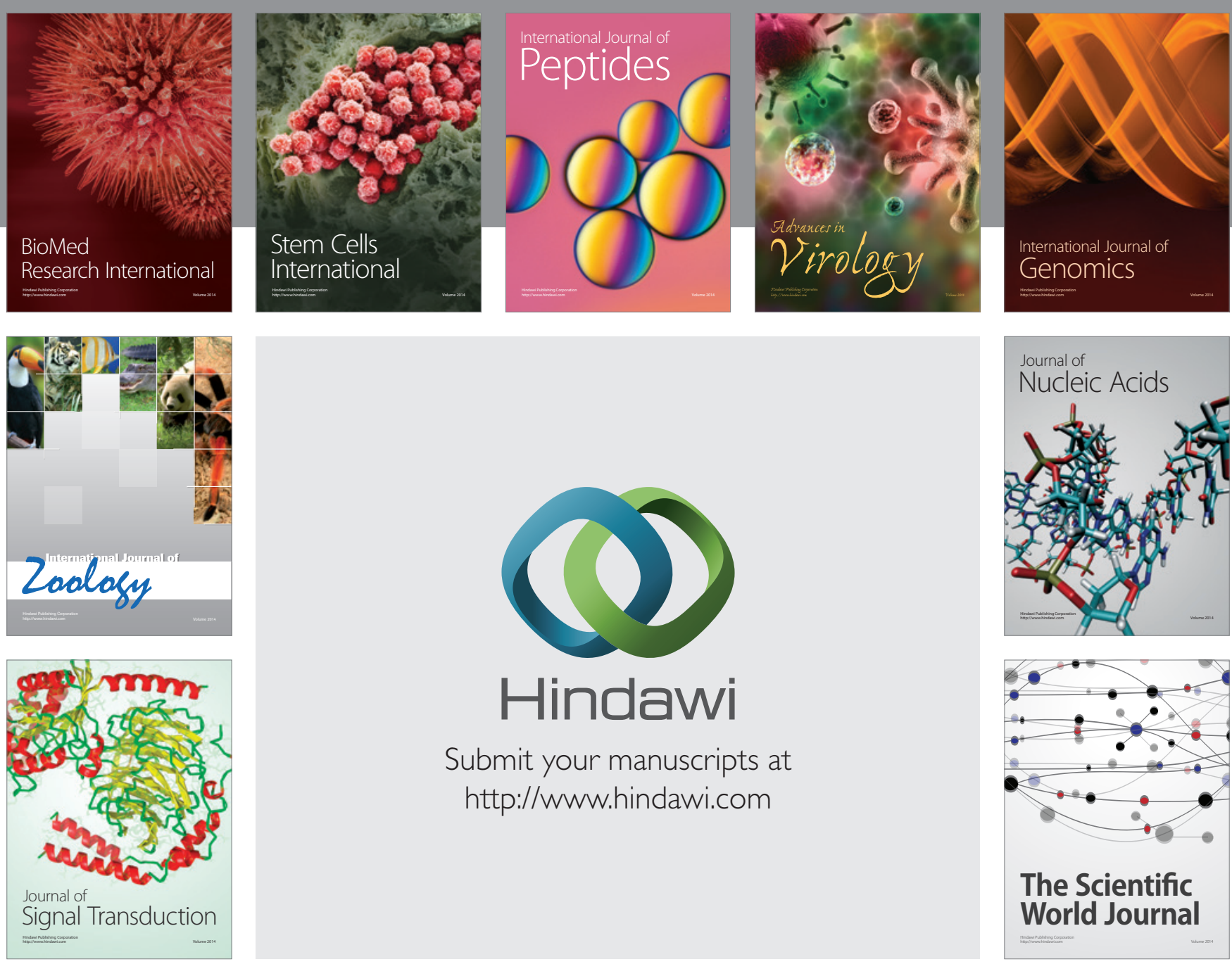

Submit your manuscripts at

http://www.hindawi.com
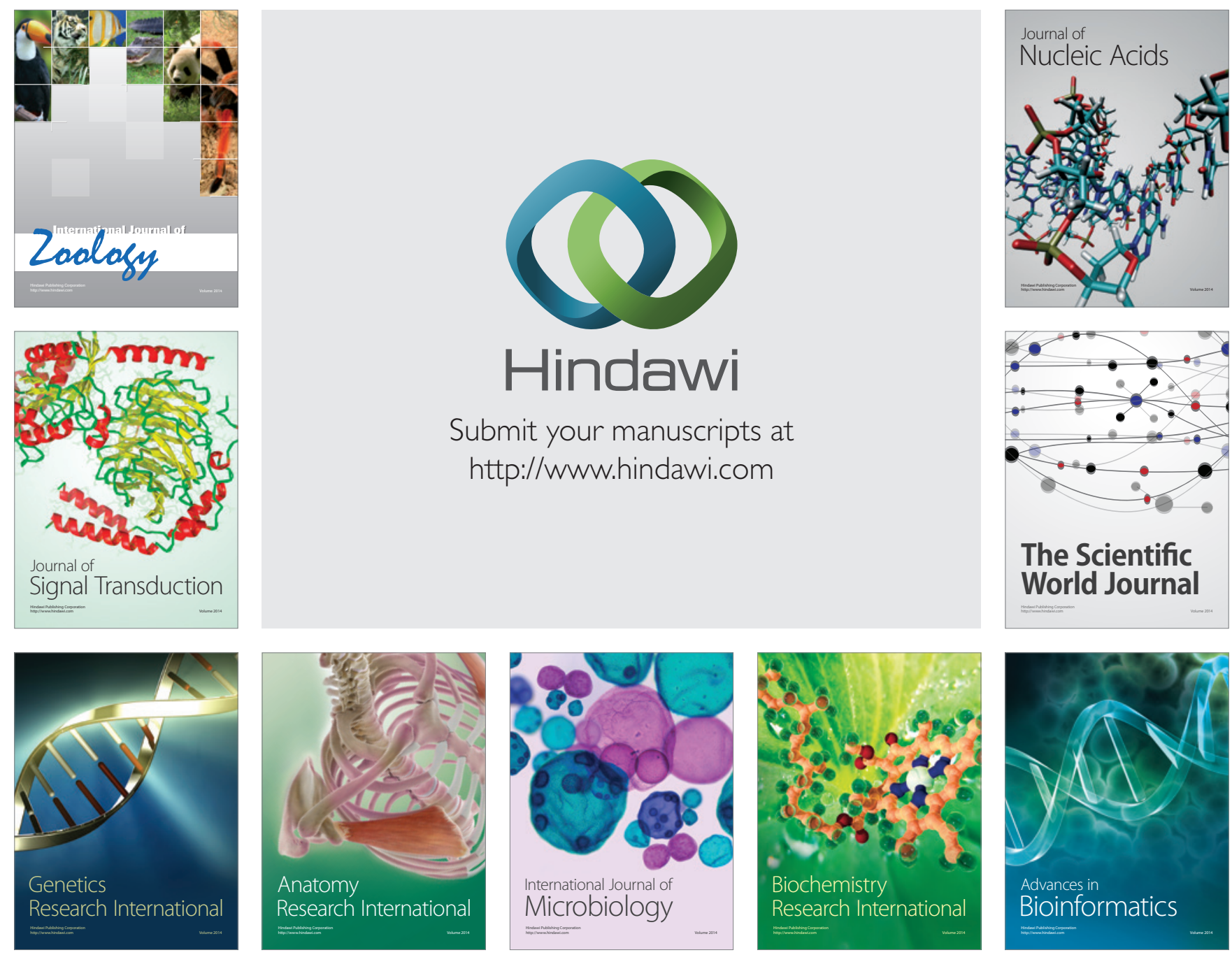

The Scientific World Journal
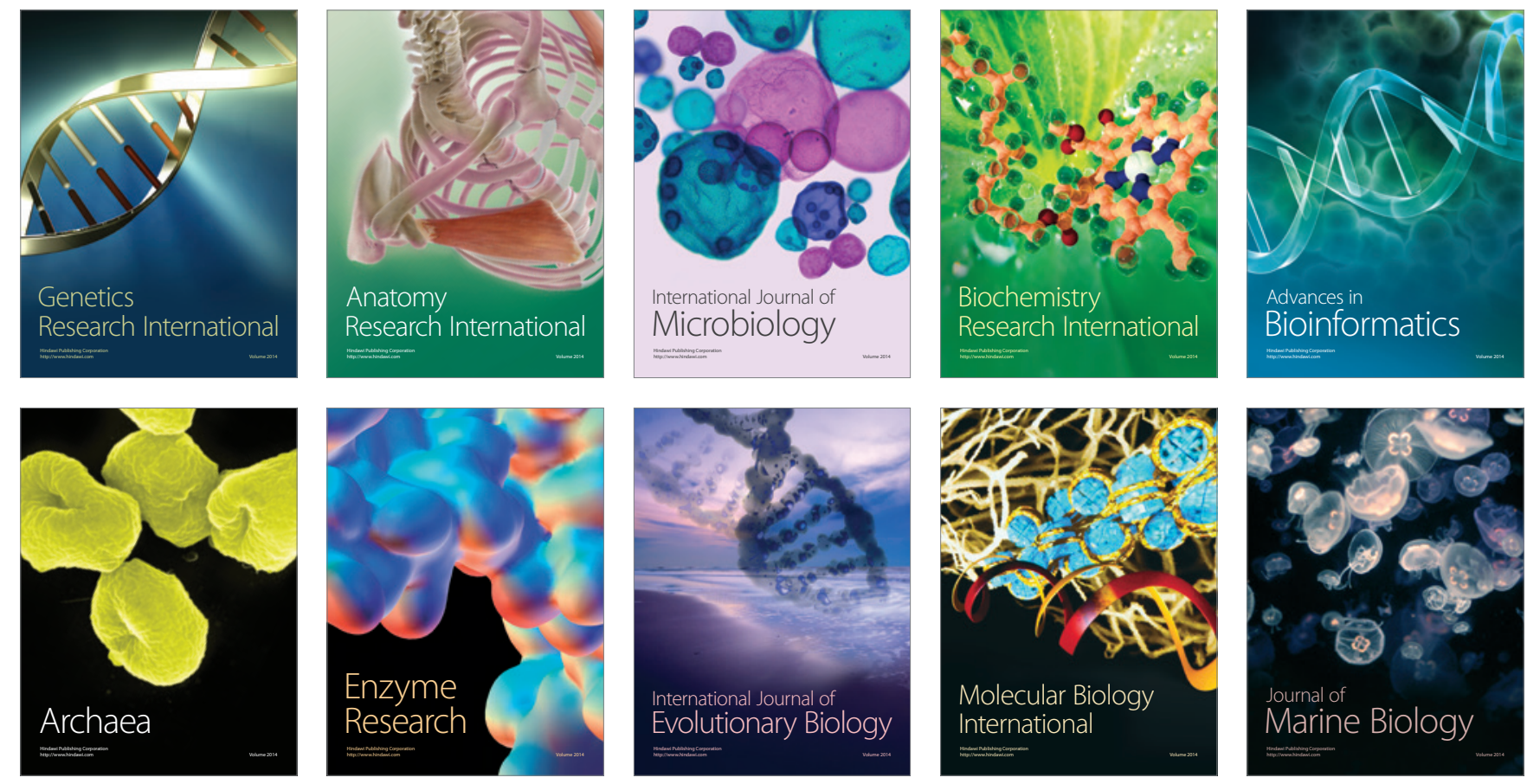